\title{
Resonances in graphene-dielectric stacks
}

\author{
Faroq Razzaz and Majeed A. S. Alkanhal
}

\begin{abstract}
Background: This paper presents a comprehensive theoretical investigation of the anomalous electromagnetic wave propagation and resonances around the trapped modes embedded in graphene dielectric stacks.

Results: Expressions for the dispersion relation, the condition of the trapped mode, the transmission coefficient, and the instantaneous field components through the graphene stack are derived. Evanescent mode coupling to the graphene-dielectric stack arises at the discrete frequencies of the trapped modes. When the wavevector is perturbed, resonances occur around the trapped modes which results in transmission anomalies and dramatic field amplification in graphene stacks.

Conclusions: Anomalous electromagnetic wave propagation and resonances in graphene-dielectric stacks are reconnoitered. The number of the conceivable discrete trapped modes and consequently the resonances in graphene-dielectric stacks can be adjusted by the graphene chemical potential.
\end{abstract}

Keywords: Graphene stacks, Resonances, Trapped modes, Transmission anomalies, Field amplification

\section{Background}

Material technology is developing with great swiftness towards constructing novel materials with tailored electromagnetic (EM) and optical properties which do not exist in nature. One of the frontiers at which major achievements are being made is graphene conception. Graphene is a monolayer of carbon atoms with massless linear carriers (electron/hole) dispersion with the effective velocity of light [1]. The special spectrum of the charge carriers leads to a number of interesting transport properties, which have been intensively studied in the literature [2]. Graphene has applications in electronics, photonics, optoelectronics, and in the development of sensory devices [3-5]. Graphene possesses a number of unique and extraordinary properties such as high charge carrier mobility, electronic energy spectrum without a gap between the conduction and valence bands, and frequency dependent absorption of electromagnetic radiation [6].

Recently, graphene attracted a great deal of attention of EM analysis and characterization $[7,8]$. Several studies were accomplished on the interaction of electromagnetic fields with graphene. EM absorbing and reflectance properties of graphene composites for different applications [9], EM wave absorption properties of graphene modified with carbon nanotube composites, and electromagnetic interference

\footnotetext{
* Correspondence: majeed@ksu.edu.sa

King Saud University, Electrical Engineering, Riyadh 11421, Saudi Arabia
}

shielding with flexible graphene/polymer composite films have been presented recently in literature. Study presented in [10] showed that approximately half of the incident EM power can be absorbed by a graphene-dielectric stack of overall thickness of two nanometers. Other studies showed that both transverse magnetic (TM) and transverse electric (TE) polarized plasmons can be supported in graphene [11]. Hyperbolic wavevector dispersion at mid- and far-infrared frequencies in multilayer graphene dielectric stacks have been studied in [12]. Waveguide modes based on multilayer graphene dielectric structure and the effective medium theory of graphene structures in infrared band have been presented in [12]. The conditions of large wavevector propagating EM waves in subwavelength periodic multilayer graphene structures have been theoretically investigated in [13].

Further notable behavior of optical waves in graphene structures have been noticed and reported by researchers. Structures that defines visual transparency of graphene is discussed in [7]. Perfect absorption in graphene multilayers and the anomalous absorption in the magneto-optical response of graphene is presented in [14]. The interaction of both interband and intraband transitions in graphene permits altering a graphene dielectric multilayer structure into an electromagnetically transparent and/or opaque medium in the $\mathrm{THz}$ or infrared frequency ranges [15]. In general, at $\mathrm{THz}$ frequencies, the spatial dispersion introduced by the periodicity of graphene can be neglected as suggested by 
[16]. Similar studies on optical and electromagnetic periodic waveguiding structures described similar wave behavior [14, $17,18]$. In quantum mechanics, the wave interaction can be debated in terms of particles quantum tunneling. This EM behavior can be utilized in many applications in photonics and optoelectronics; such as in polarization control and filtering, in photonic devices, in surface plasmon resonance, in tuning of light emitting diodes and lasers, and in total internal reflection fluorescence microscopy.

In this work, a graphene-dielectric stack in an anisotropic ambient medium is considered to examine the formation of evanescent wave coupling, discrete trapped modes formation, resonances, and the associated transmission and field anomalies in the elliptic dispersive graphene stack. The transfer matrix method (TMM) is developed to derive the transmission coefficients in both the non-resonant and the resonant states. The instantaneous field components are presented to display the field amplifications at the resonant frequencies. The effect of the graphene chemical potential on the number of the discrete trapped modes and consequently the resonances is studied as well.

\section{Formulation and analysis}

Consider the physical model shown in Fig. 1(a), where an anisotropic ambient medium is defected by a

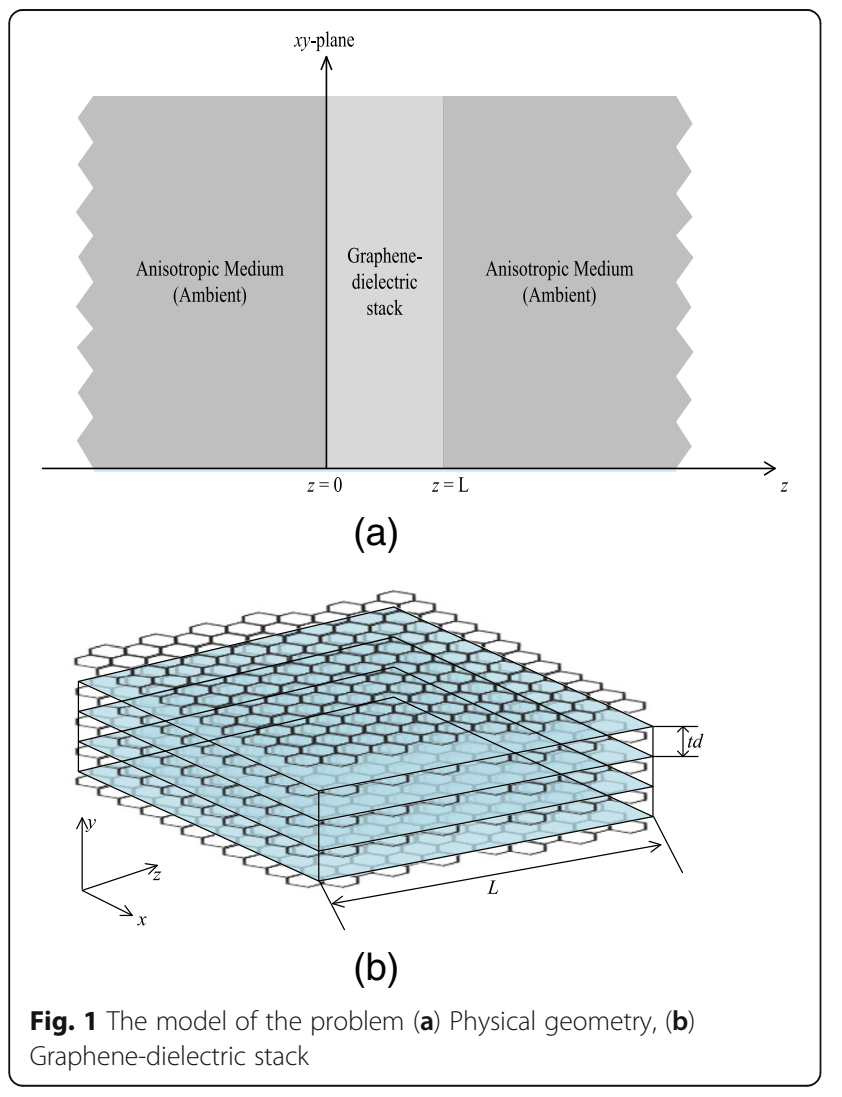

graphene-dielectric stack. The graphene-dielectric stack is finite in the $z$-direction as shown in Fig. 1(b). To investigate the evanescent wave behavior around the graphene stack, the ambient medium is designated as an anisotropic medium that can support both propagating and evanescent modes at the same parallel wavevector. The anisotropic medium is represented by the following constitutive relations

$$
\begin{aligned}
\bar{D} & =\overline{\bar{\varepsilon}} \bar{E} \\
\bar{B} & =\overline{\bar{\mu}} \bar{H}
\end{aligned}
$$

where $\overline{\bar{\varepsilon}}$ is the $3 \times 3$ permittivity tensor and $\overline{\bar{\mu}}$ is the $3 \times$ 3 permeability tensor given by

$$
\overline{\bar{\varepsilon}}=\left[\begin{array}{lll}
\varepsilon_{x x} & \varepsilon_{x y} & \varepsilon_{x z} \\
\varepsilon_{y x} & \varepsilon_{y y} & \varepsilon_{y z} \\
\varepsilon_{z x} & \varepsilon_{z y} & \varepsilon_{z z}
\end{array}\right] \text {, and } \overline{\bar{\mu}}=\left[\begin{array}{lll}
\mu_{x x} & \mu_{x y} & \mu_{x z} \\
\mu_{y x} & \mu_{y y} & \mu_{y z} \\
\mu_{z x} & \mu_{z y} & \mu_{z z}
\end{array}\right]
$$

Consider a general obliquely incident plane wave with time dependency $e^{-i \omega t}$. The fields are defined as

$$
\begin{aligned}
\mathbf{E}(x, y, z ; t) & =\mathbf{E}_{0} e^{i\left(k_{x} x+k_{y} y+k_{z} z-\omega t\right)} \\
& =\left[\begin{array}{l}
E_{x}(z) \\
E_{y}(z) \\
E_{z}(z)
\end{array}\right] e^{i\left(k_{x} x+k_{y} y-\omega t\right)} \\
\mathbf{H}(x, y, z ; t) & =\mathbf{H}_{0} e^{i\left(k_{x} x+k_{y} y+k_{z} z-\omega t\right)} \\
& =\left[\begin{array}{l}
H_{x}(z) \\
H_{y}(z) \\
H_{z}(z)
\end{array}\right] e^{i\left(k_{x} x+k_{y} y-\omega t\right)}
\end{aligned}
$$

where $\kappa=\left(k_{x}, k_{y}\right)$ is the wavevector parallel to the graphene stack and $\omega$ is the angular frequency of the incident plane wave. Using the Berreman $4 \times 4$ matrix method [19], the Maxwell equations are reduced to four ordinary differential equations in terms of tangential electric and magnetic field components.

$$
\frac{d \phi(z)}{d z}=i J A \phi(z)
$$

where $A$ is an $4 \times 4$ matrix that depends on the properties of the medium $(\overline{\bar{\varepsilon}}$ and $\overline{\bar{\mu}})$, the tangential wavevector components, and the frequency of the incident wave. It is given by

$$
A=\left[\begin{array}{ll}
a_{11} & a_{12} \\
a_{21} & a_{22}
\end{array}\right]
$$

The elements of the above matrix $A$ are given by 


$$
\begin{aligned}
a_{11}=\left[\begin{array}{ll}
\frac{\omega}{c} \varepsilon_{x x}-\frac{c}{\omega} \frac{k_{y}^{2}}{\mu_{z z}}-\frac{\omega}{c} \frac{\varepsilon_{x z} \varepsilon_{z x}}{\varepsilon_{z z}} & \frac{\omega}{c} \varepsilon_{x y}+\frac{c}{\omega} \frac{k_{x} k_{y}}{\mu_{z z}}-\frac{\omega}{c} \frac{\varepsilon_{x z} \varepsilon_{z y}}{\varepsilon_{z z}} \\
\frac{\omega}{c} \varepsilon_{y x}+\frac{c}{\omega} \frac{k_{x} k_{y}}{\mu_{z z}}-\frac{\omega}{c} \frac{\varepsilon_{y z} \varepsilon_{z x}}{\varepsilon_{z z}} & \frac{\omega}{c} \varepsilon_{y y}-\frac{c}{\omega} \frac{k_{x}^{2}}{\mu_{z z}}-\frac{\omega}{c} \frac{\varepsilon_{y z} \varepsilon_{z y}}{\varepsilon_{z z}}
\end{array}\right], & \boldsymbol{v}_{ \pm}^{a p}=\left[\begin{array}{c}
\omega \\
\frac{\omega}{c} \mu_{x a} \\
\mp k_{z}^{a p} \\
0
\end{array}\right] \\
a_{12}=\left[\begin{array}{ll}
k_{y}\left(\frac{\varepsilon_{x z}}{\varepsilon_{z z}}-\frac{\mu_{z x}}{\mu_{z z}}\right) & -k_{x} \frac{\varepsilon_{x z}}{\varepsilon_{z z}}-k_{y} \frac{\mu_{z y}}{\mu_{z z}} \\
k_{x} \frac{\mu_{z x}}{\mu_{z z}}+k_{y} \frac{\varepsilon_{y z}}{\varepsilon_{z z}} & k_{x}\left(\frac{\mu_{z y}}{\mu_{z z}}-\frac{\varepsilon_{y z}}{\varepsilon_{z z}}\right)
\end{array}\right], & \boldsymbol{v}_{ \pm}^{a e}=\left[\begin{array}{c} 
\pm k_{z}^{a e} \\
0 \\
0 \\
\frac{\omega}{c} \varepsilon_{x a}
\end{array}\right]
\end{aligned}
$$$$
a_{21}=\left[\begin{array}{cc}
k_{y}\left(\frac{\varepsilon_{z x}}{\varepsilon_{z z}}-\frac{\mu_{x z}}{\mu_{z z}}\right) & k_{x} \frac{\mu_{x z}}{\mu_{z z}}+k_{y} \frac{\varepsilon_{z y}}{\varepsilon_{z z}} \\
-k_{x} \frac{\varepsilon_{z x}}{\varepsilon_{z z}}-k_{y} \frac{\mu_{y z}}{\mu_{z z}} & k_{x}\left(\frac{\mu_{y z}}{\mu_{z z}}-\frac{\varepsilon_{z y}}{\varepsilon_{z z}}\right)
\end{array}\right] \text {, and }
$$$$
a_{22}=\left[\begin{array}{ll}
\frac{\omega}{c} \mu_{x x}-\frac{c}{\omega} \frac{k_{y}^{2}}{\varepsilon_{z z}}-\frac{\omega}{c} \frac{\mu_{x z} \mu_{z x}}{\mu_{z z}} & \frac{\omega}{c} \mu_{x y}+\frac{c}{\omega} \frac{k_{x} k_{y}}{\varepsilon_{z z}}-\frac{\omega}{c} \frac{\mu_{x z} \mu_{z y}}{\mu_{z z}} \\
\frac{\omega}{c} \mu_{y x}+\frac{c}{\omega} \frac{k_{x} k_{y}}{\varepsilon_{z z}}-\frac{\omega}{c} \frac{\mu_{y z} \mu_{z x}}{\mu_{z z}} & \frac{\omega}{c} \mu_{y y}-\frac{c}{\omega} \frac{k_{x}^{2}}{\varepsilon_{z z}}-\frac{\omega}{c} \frac{\mu_{y z} \mu_{z y}}{\mu_{z z}}
\end{array}\right]
$$

Additionally, $\phi(z)$ and $J$ are given, respectively, by

$$
\phi(z)=\left[\begin{array}{c}
E_{x}(z) \\
E_{y}(z) \\
H_{x}(z) \\
H_{y}(z)
\end{array}\right], J=\left[\begin{array}{cccc}
0 & 0 & 0 & 1 \\
0 & 0 & -1 & 0 \\
0 & -1 & 0 & 0 \\
1 & 0 & 0 & 0
\end{array}\right],
$$

and $c$ is the speed of light in vacuum.

\section{Fields in the anisotropic ambient medium}

The anisotropic ambient medium has the following properties:

$$
\overline{\bar{\varepsilon}}_{a}=\left[\begin{array}{ccc}
\varepsilon_{x a} & 0 & 0 \\
0 & \varepsilon_{y a} & 0 \\
0 & 0 & \varepsilon_{z a}
\end{array}\right], \text { and } \overline{\bar{\mu}}_{a}=\left[\begin{array}{ccc}
\mu_{x a} & 0 & 0 \\
0 & \mu_{y a} & 0 \\
0 & 0 & \mu_{z a}
\end{array}\right]
$$

These properties provide two real and two imaginary $z$-directional wavenumbers corresponding to propagating and evanescent modes. These modes are the eigenvalues of the $4 \times 4$ matrix $J A$ in the medium. At $k_{y}=0$, the $z$-directional propagating and evanescent wavenumbers in the anisotropic ambient medium are given, respectively, by

$$
\begin{aligned}
k_{z}^{a p} & = \pm \sqrt{\frac{\omega^{2}}{c^{2}} \varepsilon_{y a} \mu_{x a}-\frac{\mu_{x a}}{\mu_{z a}} k_{x}^{2}} \\
k_{z}^{a e} & = \pm \sqrt{\frac{\omega^{2}}{c^{2}} \varepsilon_{x a} \mu_{y a}-\frac{\varepsilon_{x a}}{\varepsilon_{z a}} k_{x}{ }^{2}}
\end{aligned}
$$

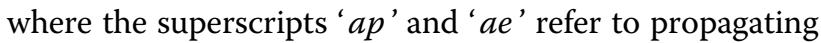
and evanescent modes in the ambient medium, respectively. The corresponding tangential field components (eigenvectors) associated with these wavenumbers are given by
The propagating $z$-directional wavenumber $\left(k_{z}^{a p}\right)$ should be real, while the evanescent $z$-directional wavenumber $\left(k_{z}^{a e}\right)$ should be imaginary. Therefore, for the anisotropic ambient medium, the elements of the permittivity tensor $\overline{\bar{\varepsilon}}_{a}$ and permeability tensor $\overline{\bar{\mu}}_{a}$ must satisfy the following conditions:

$$
\varepsilon_{y a} \mu_{z a}>\frac{k_{x}^{2}}{\left(\frac{\omega}{c}\right)^{2}}, \mu_{y a} \varepsilon_{z a}<\frac{k_{x}^{2}}{\left(\frac{\omega}{c}\right)^{2}}
$$

which implies that $\varepsilon_{y a} \mu_{z a}>\mu_{y a} \varepsilon_{z a}$.

\section{Fields in the graphene-dielectric stack}

The electromagnetic properties of graphene are described in terms of the surface conductivity $\sigma$. Kubo model of conductivity is used for the graphene layer that takes into account the interband and intraband transitions and is given by [12]

$$
\sigma=\sigma_{\text {inter }}+\sigma_{\text {intra }}
$$

where the intraband and interband conductivity are given, respectively, by

$$
\begin{aligned}
\sigma_{\text {intra }} & =\frac{i 4 e^{2} k_{B} T}{\hbar(\omega+i / \tau)}\left(\frac{\mu_{c}}{k_{B} T}+2 \ln \left(e^{-\mu_{c} / k_{B} T}+1\right)\right) \\
\sigma_{\text {inter }} & =\frac{i 4 e^{2} k_{B} T}{\hbar} \int_{0}^{\infty} \frac{f_{d}(-E)-f_{d}(E)}{(\omega+i / \tau)^{2}-4(E / \hbar)^{2}} d E \\
& \approx \frac{i e^{2}}{\hbar^{2}} \ln \left(\frac{2\left|\mu_{c}\right|-(\omega+i / \tau) \hbar}{2\left|\mu_{c}\right|+(\omega+i / \tau) \hbar}\right)
\end{aligned}
$$

where $\mu_{c}$ is the chemical potential, $\omega$ is the angular frequency, $k_{B}$ is the Boltzmann's constant, $\hbar$ is the reduced Planck constant, $T$ is the temperature (in Kelvin), $e$ is the charge of the electron, $\tau$ is the relaxation time, $E$ is the Fermi energy, and $f_{d}(E)$ is the Fermi-Dirac distribution given by $f_{d}(E)=1 /\left(e^{(E-\mu c) / k_{B} T}+1\right)$.

Since the graphene carriers are well localized within one-atom thick layer ( $x z$-plane), the relative anisotropic permittivity of graphene can be characterized as [14] 


$$
\varepsilon_{g}=\left[\begin{array}{ccc}
\varepsilon_{\|} & 0 & 0 \\
0 & \varepsilon_{\perp} & 0 \\
0 & 0 & \varepsilon_{\|}
\end{array}\right]
$$

where $\varepsilon_{\|}$and $\varepsilon_{\perp}$ denote the parallel and normal components of the permittivity of the graphene layer, respectively. The parallel permittivity can be determined from the following expression [12]

$$
\varepsilon_{\|}=1+i \frac{\sigma}{\omega \varepsilon_{0} t_{g}}
$$

where $t_{g}$ is the thickness of the graphene layer. Since graphene is a 2D material, the normal fields cannot excite any current in the graphene layer. Then, the normal permittivity $\varepsilon_{\perp}=1$.

The equivalent effective permittivity tensor of the graphene-dielectric stack shown in Fig. 1(b) can be determined using the effective medium theory (EMT) [12] as

$$
\begin{aligned}
\varepsilon_{s} & =\left[\begin{array}{ccc}
\varepsilon_{x s} & 0 & 0 \\
0 & \varepsilon_{y s} & 0 \\
0 & 0 & \varepsilon_{z s}
\end{array}\right] \\
\varepsilon_{x s} & =\varepsilon_{z s}=f \varepsilon_{\|}+(1-f) \varepsilon_{d} \\
\varepsilon_{y s} & =\frac{\varepsilon_{\perp} \varepsilon_{d}}{f \varepsilon_{d}+(1-f) \varepsilon_{\perp}}
\end{aligned}
$$

where $\varepsilon_{d}$ is the permittivity of the dielectric and $f$ is the fill fraction of the graphene defined as

$$
f=\frac{t_{g}}{t_{g}+t_{d}}
$$

The $z$-directional propagating and evanescent wavenumbers in the graphene-dielectric stack when $k_{y}=0$ are given, respectively, by

$$
\begin{aligned}
& k_{z}^{s p}= \pm \sqrt{\frac{\omega^{2}}{c^{2}} \varepsilon_{x s}-k_{x}^{2}} \\
& k_{z}^{s e}= \pm \sqrt{\frac{\omega^{2}}{c^{2}} \varepsilon_{y s}-k_{x}^{2}}
\end{aligned}
$$

where the superscripts ' $s p$ ' and ' $s e$ ' refer to the propagating and evanescent modes in the graphene-dielectric stack respectively. The corresponding field components associated with these wavenumbers are given by the eigenvectors

$$
\boldsymbol{v}_{ \pm}^{s p}=\left[\begin{array}{c} 
\pm k_{z}^{s p} \\
0 \\
0 \\
\frac{\omega}{c} \varepsilon_{x s}
\end{array}\right]
$$

$$
\boldsymbol{v}_{ \pm}^{s e}=\left[\begin{array}{c}
0 \\
-\frac{\omega}{c} \\
\pm k_{z}^{s e} \\
0
\end{array}\right]
$$

The propagating $z$-directional wavenumber $\left(k_{z}^{s p}\right)$ should be real while the evanescent $z$-directional wavenumber $\left(k_{z}^{s e}\right)$ should be imaginary. Therefore, for the graphene-dielectric stack, the elements of the permittivity tensor $\overline{\bar{\varepsilon}}_{s}$ must satisfy the following conditions:

$$
\left|\varepsilon_{x s}\right|>\frac{k_{x}^{2}}{\left(\frac{\omega}{c}\right)^{2}},\left|\varepsilon_{y s}\right|<\frac{k_{x}^{2}}{\left(\frac{\omega}{c}\right)^{2}}
$$

which implies that $\left|\varepsilon_{x s}\right|>\left|\varepsilon_{y s}\right|$.

The trapped modes in the slab are characterized by $k_{z}^{s p, e}=0 \quad$ (no $z$-directional propagation) and their frequencies belong to the continuous interval $I$ bounded by the dispersion curves of the propagating and evanescent waves of the stack given by Eqs. (19) and (20) respectively, i.e. $k_{x} /\left(\sqrt{\mid \varepsilon_{x s}} \mid\right) \leq I \leq k_{x} /\left(\sqrt{\left|\varepsilon_{y s}\right|}\right)$. Evanescent wave coupling arises when the incident frequencies belong to the continuous spectrum interval $I$ and the tangential field components of the anisotropic ambient evanescent modes match those of the propagating modes in the graphene-dielectric stack medium. If the normalized parallel wavevector is perturbed, the resonance around the trapped modes will occur around the discrete trapped modes. The construction of the trapped modes can be determined by matching the evanescent fields in the ambient medium with the propagating fields in the graphene-dielectric stack, so that

$$
\begin{aligned}
& {\left[\begin{array}{c}
E_{x} \\
E_{y} \\
H_{x} \\
H_{y}
\end{array}\right]=C_{1}\left[\begin{array}{c}
k_{z}^{a e} \\
0 \\
\omega^{0} \\
\frac{\omega}{c} \varepsilon_{x a}
\end{array}\right] e^{-i k_{z}^{a e} z}} \\
& =C_{2}\left[\begin{array}{c}
k_{z}^{s p} \\
0 \\
0 \\
\frac{\omega}{c} \varepsilon_{x s}
\end{array}\right] e^{i k_{z}^{s p} z}+C_{3}\left[\begin{array}{c}
-k_{z}^{s p} \\
0 \\
0 \\
\frac{0}{c} \varepsilon_{x s}
\end{array}\right] e^{-i k_{z}^{s p} z} \quad(0<z<L) \\
& =C_{4}\left[\begin{array}{c}
k_{z}^{a e} \\
0 \\
0 \\
\frac{\omega}{c} \varepsilon_{x a}
\end{array}\right] e^{i k_{z}^{a e}(z-L)} \\
& (z>, L)
\end{aligned}
$$

where $C_{1}, C_{2}, C_{3}$, and $C_{4}$ are constants that can be determined by applying the correct boundary conditions at the interfaces. The continuity of the tangential field components at the interfaces $(z=0, z=L)$ yields the following: 


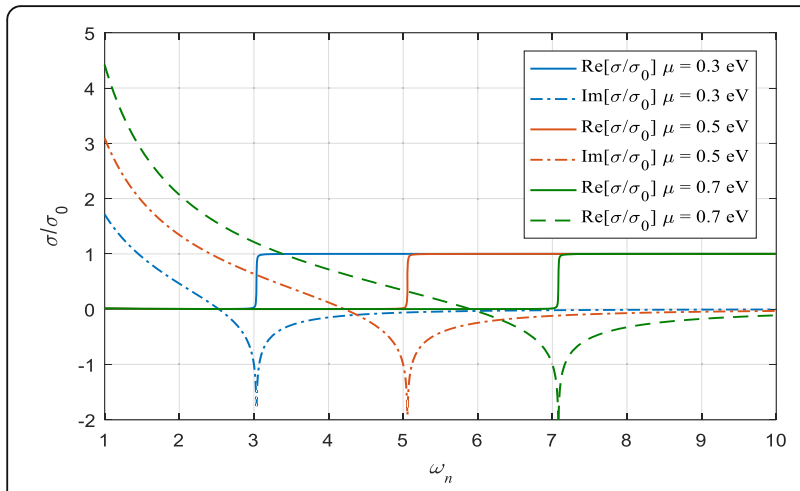

Fig. 2 Normalized Conductivity of Graphene $\left(\sigma_{0}=e^{2} /(4 \hbar)\right)$

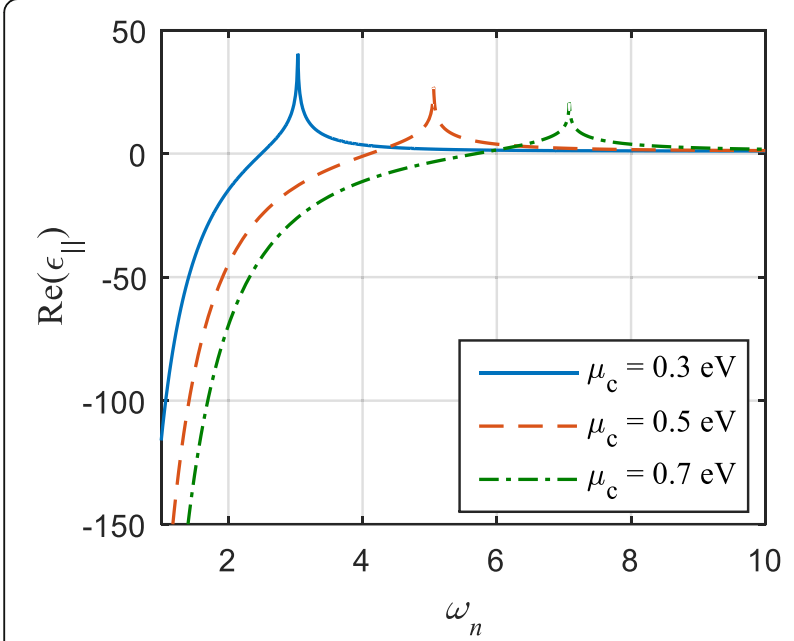

(a)

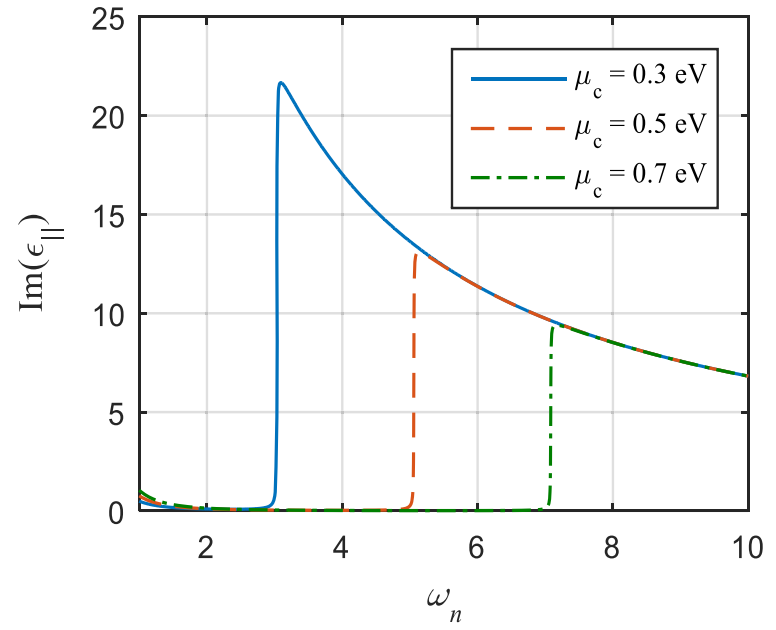

(b)

Fig. 3 Permittivity $\varepsilon_{\|}$of graphene. a real part, $\mathbf{b}$ imaginary part

$$
\left[\begin{array}{cccc}
-k_{z}^{a e} & -k_{z}^{s p} & k_{z}^{s p} & 0 \\
\varepsilon_{x a} & -\varepsilon_{x s} & -\varepsilon_{x s} & 0 \\
0 & k_{z}^{s p} e^{i k_{z}^{s p} L} & -k_{z}^{s p} e^{-i i z_{L}^{s p}} & -k_{z}^{a e} \\
0 & \varepsilon_{x s} e^{i k_{z}^{s p} L} & \varepsilon_{x s} e^{-i k_{z}^{s p} L} & -\varepsilon_{x a}
\end{array}\right]\left[\begin{array}{c}
C_{1} \\
C_{2} \\
C_{3} \\
C_{4}
\end{array}\right]=0
$$

For the above matrix to have non-trivial solution, its determinant must be equal to zero. Then, the condition of trapped modes is given by

$$
2 \cos \left(k_{z}^{s p} L\right)-i\left(\frac{k_{z}^{a e}}{k_{z}^{s p}} \frac{\varepsilon_{x s}}{\varepsilon_{x a}}+\frac{k_{z}^{s p}}{k_{z}^{a e}} \frac{\varepsilon_{x a}}{\varepsilon_{x s}}\right) \sin \left(k_{z}^{s p} L\right)=0
$$

The incident fields hit the graphene-dielectric stack at $z=0$; hence, part of this field will be reflected whereas

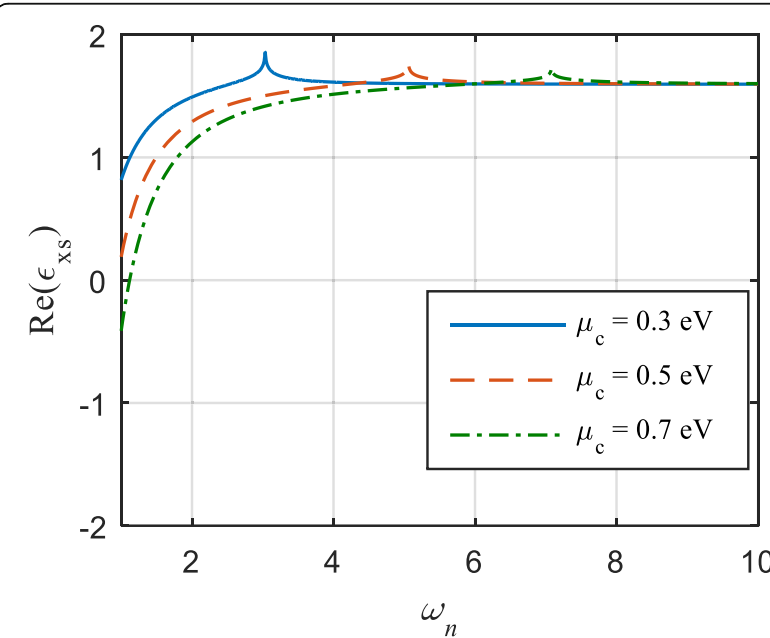

(a)

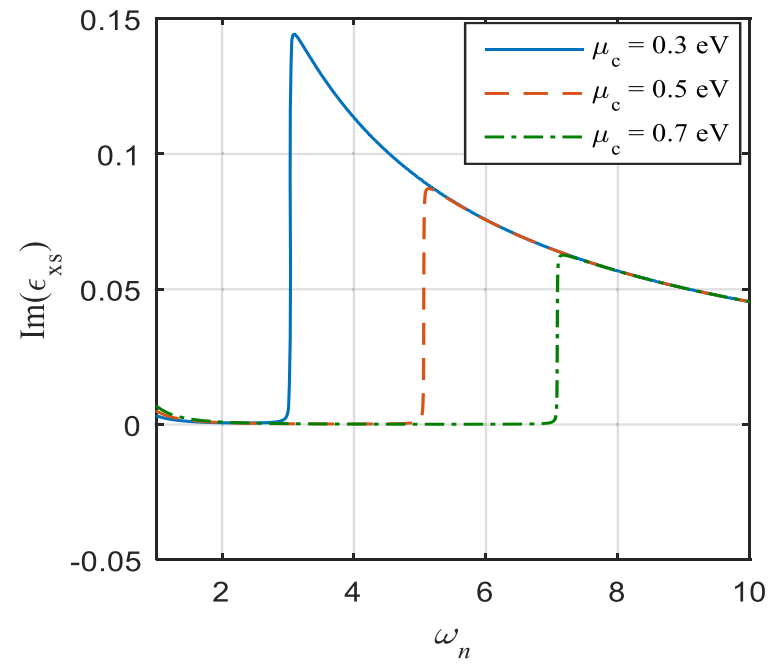

(b)

Fig. 4 Effective permittivity $\varepsilon_{x s}$ of the graphene-dielectric stack. a real part, $\mathbf{b}$ imaginary part 
the other part will be transmitted to the other side. Therefore, the resulting fields outside the graphenedielectric stack will be as follows:

$$
\psi(z)=\left\{\begin{array}{cc}
v_{+}^{a p} e^{i k_{z}^{a p} z}+r^{p}{ }^{a p p} e^{-i k_{z}^{a p} z}+r_{-}^{e} v^{a e} e^{-i k_{z}^{a z z} z} & (z<0) \\
t_{+}^{p} v_{+}^{a p} e^{i k_{z}^{a p}}(z-L)+t_{+}^{e} v_{+}^{a e} e^{i k_{z}^{e}(z-L)} & (z>L)
\end{array}\right.
$$

where, $v_{+}^{a p} e^{i k_{z}^{a p}} z$ is the incident field, $v_{ \pm}^{a p, e}$ are the eigenvectors associated with the eigenvalues ( $z$-directional wavenumbers) $\pm k_{z}^{a p, e}$ in the anisotropic ambient medium, and $r_{-}^{p, e}$ and $t_{+}^{p, e}$ are the amplitudes of the reflected and transmitted fields respectively.

The tangential field components at the interfaces of the graphene-dielectric stack are continuous, so the field inside the stack can be found using the transfer matrix method (TMM) [19] as

$$
\begin{aligned}
& \psi(L)=T(0, L) \psi(0) \\
& t_{+}^{p} v_{+}^{a p}+t_{+}^{e} v_{+}^{a e}=T(0, L)\left(v_{+}^{a p}+r_{-}^{p} v_{-}^{a p}+r_{-}^{e} v_{-}^{a e}\right)
\end{aligned}
$$

where $T(0, L)=e^{i J A_{s} L}$ is the transfer matrix of the graphene stack. The transmission and reflection coefficients can be determined directly from Eq. (29).

\section{Results and discussions}

In this section, the previous derived analytical formulations are used to determine the dispersion relations, trapped mode condition, transmission coefficients in both the non-resonance and the resonance states, and the field anomalies and amplifications at the resonance frequencies through the graphene stack.

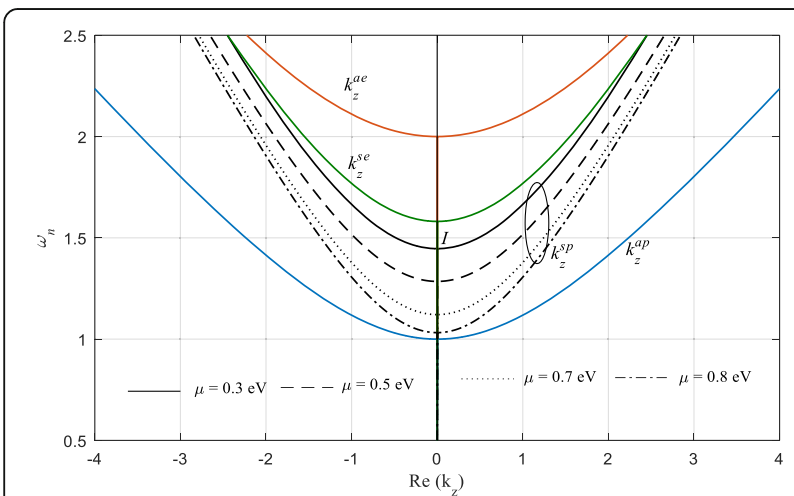

Fig. 5 Dispersion relation in the graphene stack. The blue and red lines are for the ambient medium propagating and evanescent $z-$ directional wavenumber respectively. The green line is the evanescent $z$-directional wavenumber of the graphene-dielectric stack. The black lines represent the propagating $z$-directional wavenumber of the graphene-dielectric stack for four different values of chemical potential. The interval / is bounded by the z-directional wavenumbers of the graphene-dielectric stack

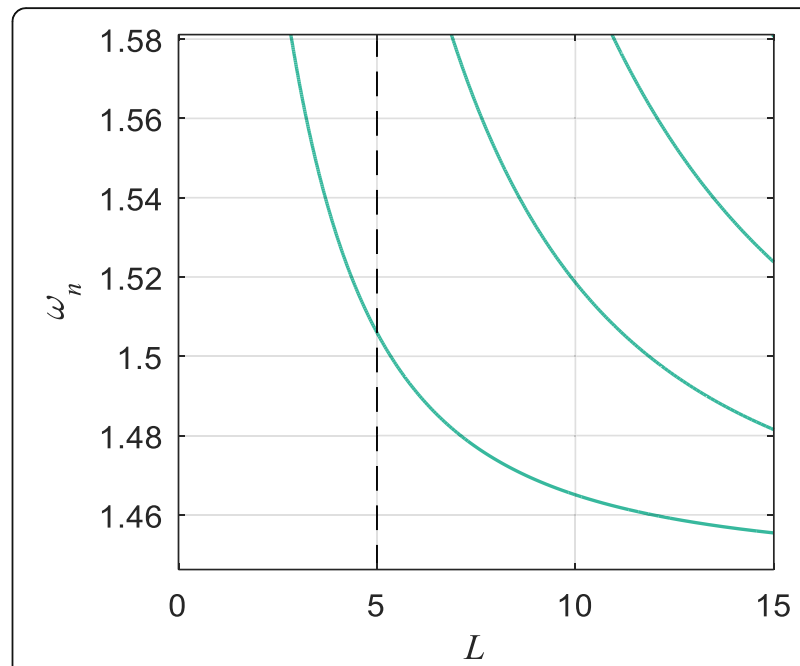

(a)

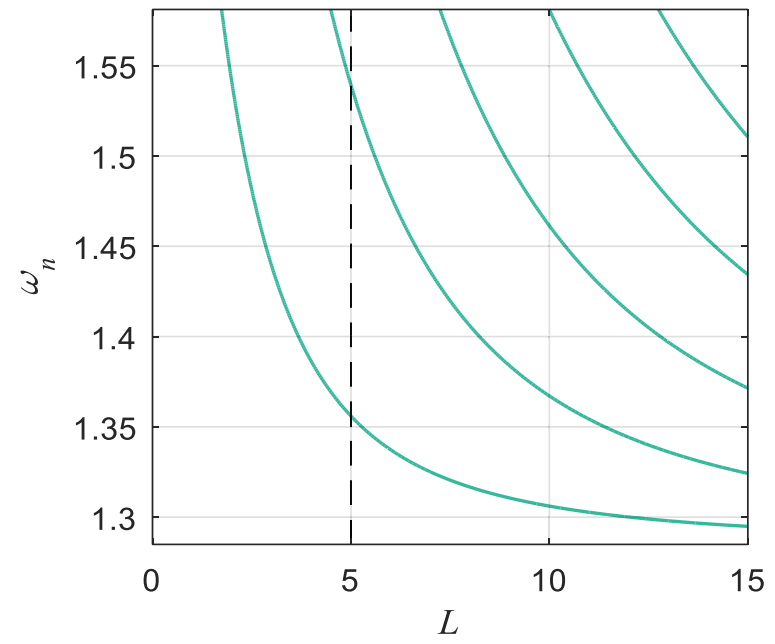

(b)

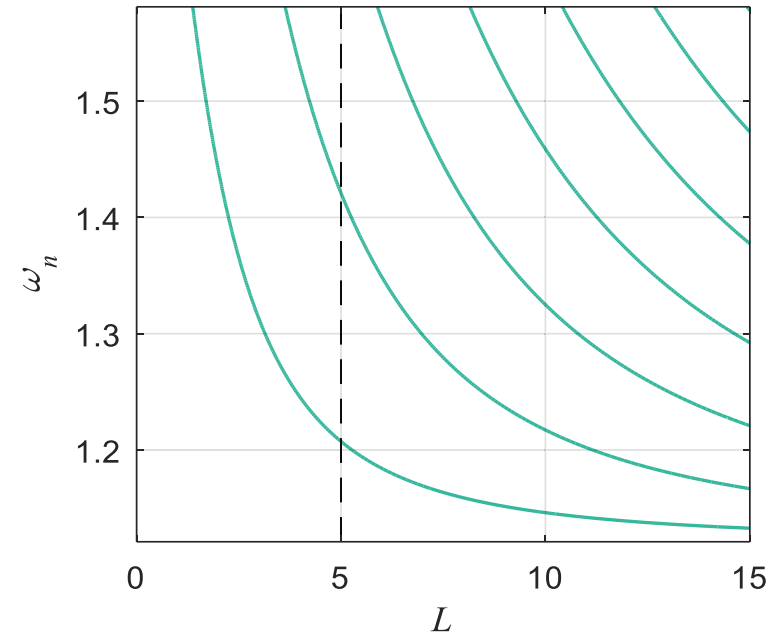

(c)

Fig. 6 Trapped modes of the graphene-dielectric stack for (a) $\mu_{c}=$ $0.3 \mathrm{eV}$, (b) $\mu_{c}=0.5 \mathrm{eV}$, and (c) $\mu_{c}=0.7 \mathrm{eV}$ 
Consider an ambient medium with the following properties

$$
\overline{\bar{\varepsilon}}_{a}=\left[\begin{array}{ccc}
2.2 & 0 & 0 \\
0 & 4 & 0 \\
0 & 0 & 1
\end{array}\right], \overline{\bar{\mu}}_{a}=\left[\begin{array}{lll}
1 & 0 & 0 \\
0 & 1 & 0 \\
0 & 0 & 1
\end{array}\right]
$$

and a graphene-dielectric stack with the properties: thickness of the graphene sheet is $0.335 \mathrm{~nm}$ and the dielectric layer is $50 \mathrm{~nm}$ with a dielectric constant $\varepsilon_{d}=1.6$.

The complex conductivity of the graphene sheet and its equivalent permittivity against the normalized frequency for different values of the chemical potential are shown, respectively, in Fig. 2 and in Fig. $3\left(\omega_{n}=\omega a / 2 \pi c\right.$, $a=10^{-6}$ meter (scaling length factor), $\omega$ is the actual frequency). The real and imaginary components of the permittivity tensor elements of the graphene-dielectric stack are shown in Fig. 4(a) and in Fig. 4(b) respectively. In the elliptic wavevector dispersion model considered here, the frequency interval is chosen to yield positive components of the effective permittivity tensor.

The dispersion relation between the normalized angular frequency $\omega_{n}$ and the real $z$-directional component of

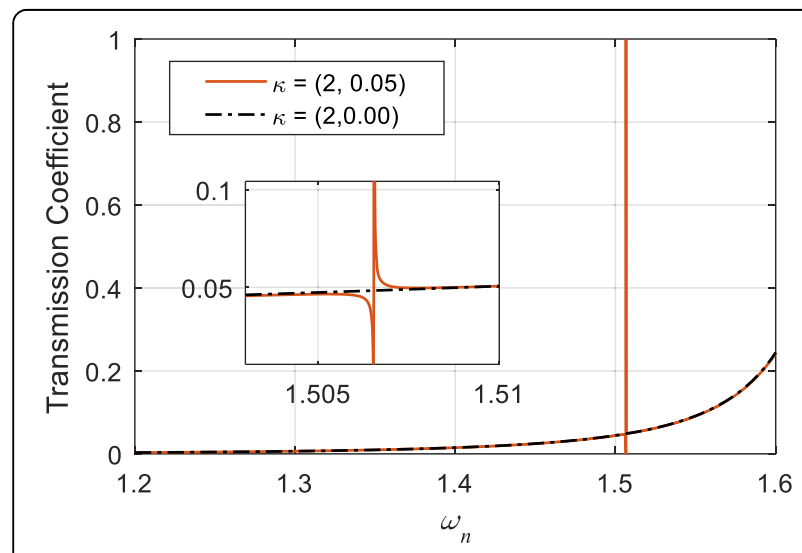

(a)

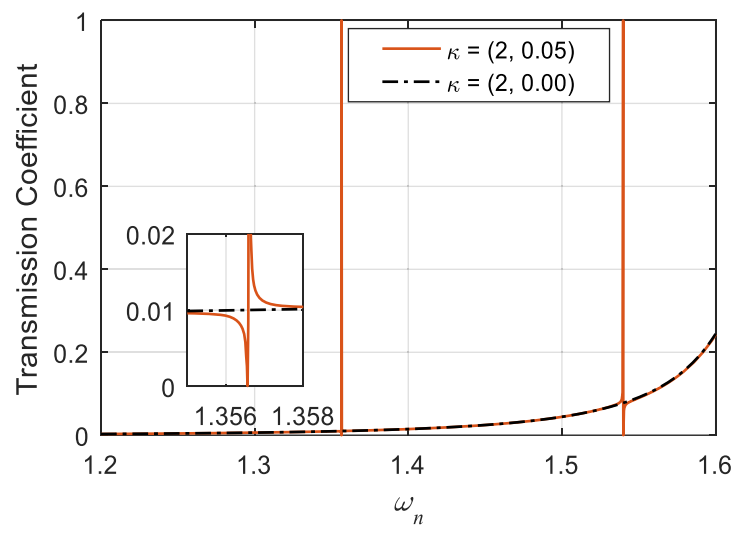

(b)

Fig. 7 Transmission Coefficient for (a) $\mu_{c}=0.3 \mathrm{eV},(\mathbf{b}) \mu_{c}=0.5 \mathrm{eV}$ the wavevector when the parallel wavevector $\kappa=\left(k_{x}, k_{y}\right)$ $=(2,0)$ is shown in Fig. 5 for four different values of the chemical potential $\mu_{c}=0.3,0.5,0.7$, and $0.8 \mathrm{eV}$. It is clearly shown that the continuous band $I$ where the trapped modes are embedded expands with the growing chemical potential. The number of the trapped modes and their frequencies, shown in Fig. 6 for the same stack thickness, adjusts with the growing chemical potential. For example, when the chemical potential is set to $0.3 \mathrm{eV}$, there is a single trapped mode at $\omega_{n}=$ $1.505988(451.7964 \mathrm{THz})$, but there are two trapped modes when the chemical potential is increased to $0.5 \mathrm{eV}$, that are located at $\omega_{n}=1.355999$ (406.7997 $\mathrm{THz})$ and $\omega_{n}=1.539336(461.8008 \mathrm{THz})$, for the same thickness of the graphene dielectric stack with 5 units in length $(L=5 \mu \mathrm{m})$. Nearby the trapped modes, the evanescent fields in the ambient medium are fitted to couple to the graphene-dielectric stack as propagating waves.

Figure 7 illustrates the transmission coefficient across the graphene-dielectric stack when its thickness is equal to 5 units in length. When the parallel wavevector is set to $(2,0)$, the graphene-dielectric

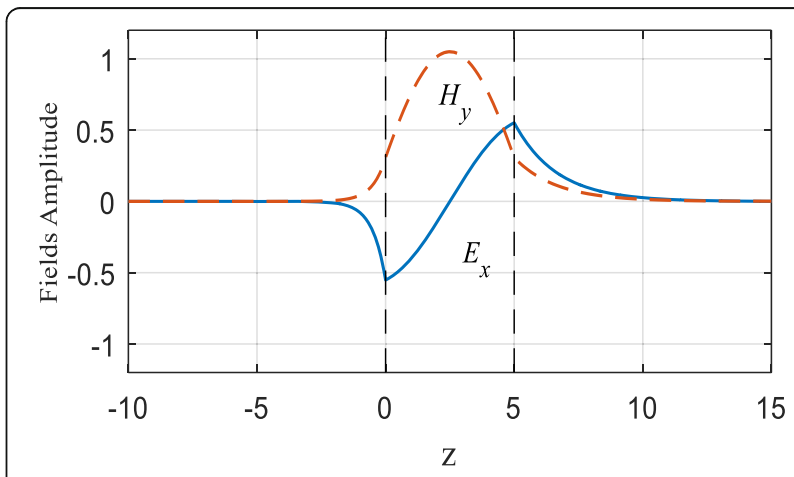

(a)

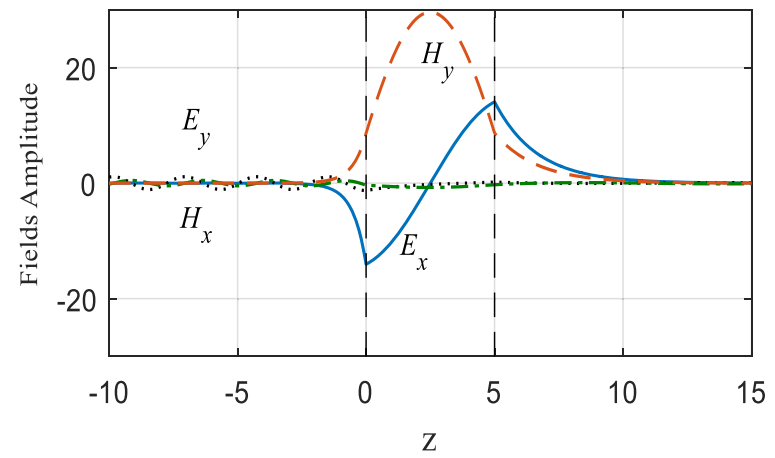

(b)

Fig. 8 Instantaneous field components for $\mu_{c}=0.3 \mathrm{eV}$. a at trapped mode (mode 0) $\omega_{n}=1.505988$ and $k=(2,0.00)$, $\mathbf{b}$ at resonance $\omega_{n}=1.506545$ and $k=(2,0.05)$ 
stack admits a single trapped mode and two trapped modes when $\mu_{c}=0.300 \mathrm{eV}$ and $\mu_{c}=0.500 \mathrm{eV}$, respectively. Moreover, when the parallel wavevector is perturbed to $(2,0.05)$, resonances around these trapped modes occur. These resonances are characterized by sharp transmission anomalies (total transmission and total reflection), and dramatic field amplification.

Figure 8(a), depicts the instantaneous fields at the trapped mode when the chemical potential is $\mu_{c}=$ $0.3 \mathrm{eV}$. The evanescent fields in the anisotropic ambient medium couple as propagating waves in the graphene stack. At resonance, the field amplification (associated with resonance) around the trapped mode frequencies is shown in Fig. 8(b). When the chemical potential is increased to $\mu_{c}=0.5 \mathrm{eV}$, the instantaneous fields at the two trapped modes supported by the stack are shown in Figs. 9(a) and 10(a). Fields amplifications at resonances around these trapped modes are shown in Figs. 9(b) and 10(b).

\section{Conclusions}

This paper develops a structured theoretical examination of the anomalous propagation and resonances

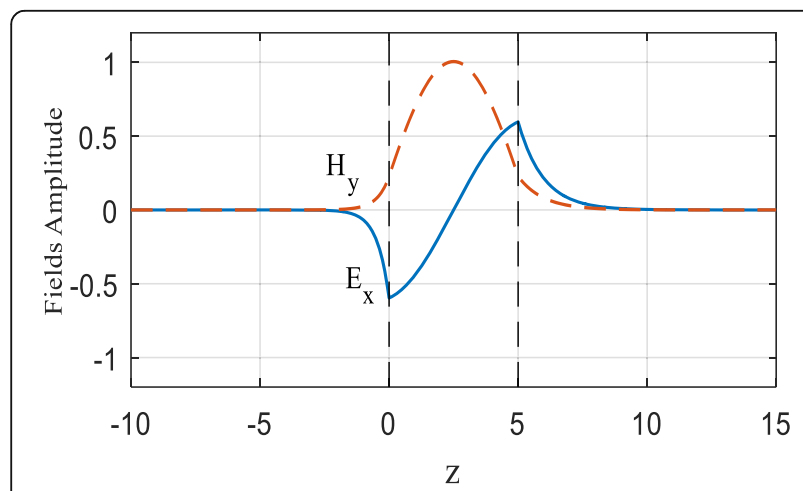

(a)

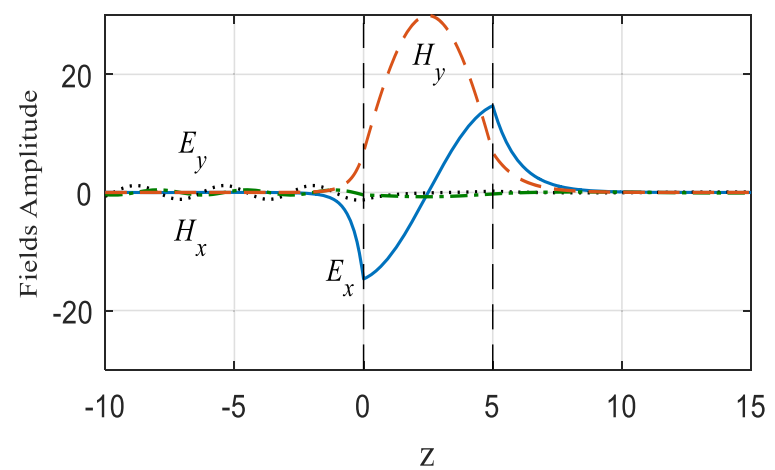

(b)

Fig. 9 Instantaneous field components for $\mu_{c}=0.5 \mathrm{eV}$. $\mathbf{a}$ at trapped mode (mode 0) $\omega_{n}=1.355999$ and $k=(2,0.00)$, $\mathbf{b}$ at resonance $\omega_{n}=$ 1.356596 and $k=(2,0.05)$

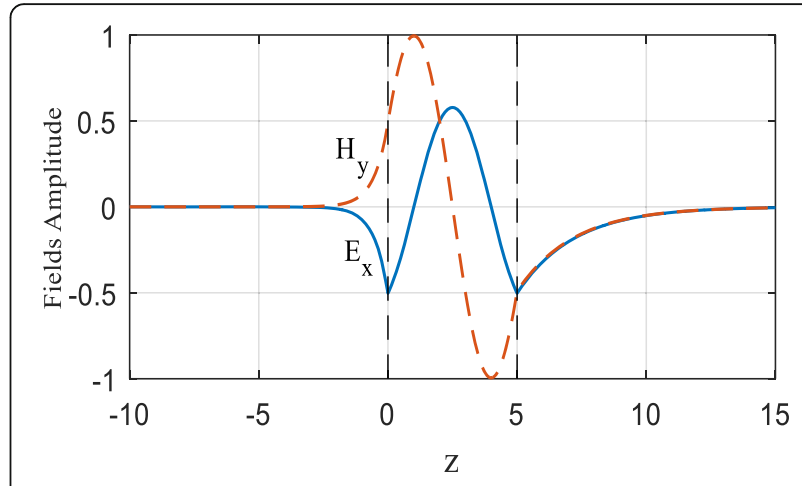

(a)

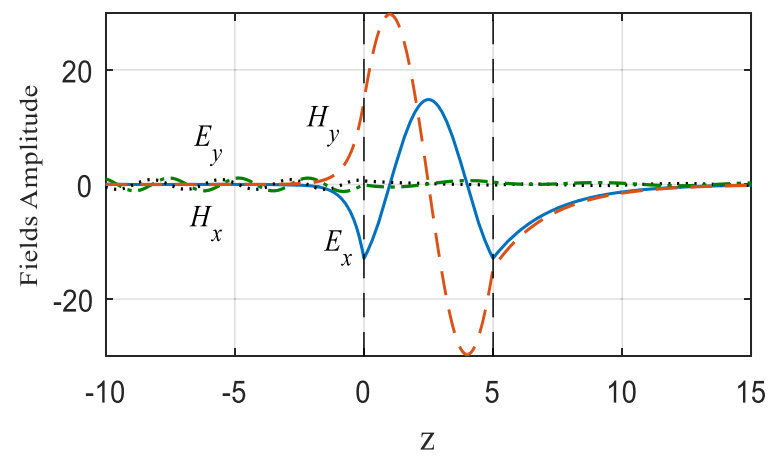

(b)

Fig 10 Instantaneous field components for $\mu_{c}=0.5 \mathrm{eV}$. $\mathbf{a}$ at trapped mode (mode 1) $\omega_{n}=1.541195$ and $k=(2,0.00)$, $\mathbf{b}$ at resonance $\omega_{n}=$ 1.5391 and $k=(2,0.05)$

of electromagnetic fields in graphene-dielectric stacks. The dispersion relations, trapped mode condition, and the propagating and evanescent modes in the graphene stack have been derived and quantified numerically. The graphene-dielectric stack can support propagating and evanescent eigen-modes at the same frequency and parallel wavevector. The evanescent modes supported in the ambient medium feasibly couple as propagating modes in the graphenedielectric stack medium at the discrete frequencies of the embedded trapped modes in the dispersive graphene. Field resonances with sharp transmission/reflection anomalies and with intense amplification in the graphene stack can occur by perturbing the parallel wavevector of the incident field around the discrete trapped modes frequencies. The chemical potential of the graphene has a noticeable effect on the width of the continuous interval where the trapped modes are embedded. The number of the trapped modes and then the discrete resonance frequencies can be controlled by changing the chemical potential of the dispersive graphene and the thickness of the stack. 


\section{Abbreviations}

EM: Electromagnetic; TE: Transverse electric; TM: Transverse magnetic; TMM: Transfer matrix method

\section{Acknowledgements}

The authors would like to express their appreciation to the Deanship of Scientific Research (DSR) at King Saud University for its funding of this research through the Research-Group no RG-1436-001.

\section{Funding}

King Saud University, Deanship of Scientific Research (DSR), Research-Group no RG-1436-001.

\section{Authors' contributions}

Both authors contributed equally to this work. Both authors read and approved the final manuscript.

\section{Competing interests}

According to the SpringerOpen's guidance on competing interests I have included this statement to indicate that none of the authors have any competing interests in the manuscript. Corresponding Author: Professor Majeed Alkanhal.

\section{Ethics approval and consent to participate}

Not applied, because this study does not involve human participants, data, tissue or animals.

Received: 5 August 2016 Accepted: 20 January 2017

Published online: 28 February 2017

\section{References}

1. Mikhailov, S., Ziegler, K.: New electromagnetic mode in graphene. Phys. Rev. Lett. 99, 016803 (2007)

2. Ziegler, K.: Robust transport properties in graphene. Phys. Rev. Lett. 97 266802 (2006)

3. Avouris, P., Freitag, M.: Graphene photonics, plasmonics, and optoelectronics. IEEE J. Sel. Top. Quantum Electron. 1, 6000112 (2014)

4. Hua-Qiang, W., Chang-Yang, L., Hong-Ming, L., He, Q.: Graphene applications in electronic and optoelectronic devices and circuits. Chinese Physics B 22 098106 (2013)

5. Kuila, T., Bose, S., Khanra, P., Mishra, A.K., Kim, N.H., Lee, J.H.: Recent advances in graphene-based biosensors. Biosens. Bioelectron. 26, 4637-4648 (2011)

6. Grushin, A.G., Valenzuela, B., Vozmediano, M.A.: Effect of Coulomb interactions on the optical properties of doped graphene. Phys. Rev. B 80, 155417 (2009)

7. Nair, R.R., Blake, P., Grigorenko, A.N., Novoselov, K.S., Booth, T.J., Stauber, T., Peres, N.M., Geim, A.K.: Fine structure constant defines visual transparency of graphene. Science 320, 1308-1308 (2008)

8. Gusynin, V., Sharapov, S., Carbotte, J.: Anomalous absorption line in the magneto-optical response of graphene. Phys. Rev. Lett. 98, 157402 (2007)

9. He, X., Zhong, X., Lin, F., Shi, W.: Investigation of graphene assisted tunable terahertz metamaterials absorber. Optical Materials Express 6, 331-342 (2016)

10. D'Aloia, A., Marra, F., Tamburrano, A., De Bellis, G., Sarto, M.S.: Electromagnetic absorbing properties of graphene-polymer composite shields. Carbon 73, 175-184 (2014)

11. Batrakov, K., Kuzhir, P., Maksimenko, S., Paddubskaya, A., Voronovich, S., Lambin, P., Kaplas, T., Svirko, Y.: Flexible transparent graphene/polymer multilayers for efficient electromagnetic field absorption. Sci. Rep. 4, 7191 (2014)

12. Koppens, F.H., Chang, D.E., Garcia de Abajo, F.J.: Graphene plasmonics: a platform for strong light-matter interactions. Nano Lett. 11, 3370-3377 (2011)

13. Zhu, B., Ren, G., Zheng, S., Lin, Z., Jian, S.: Nanoscale dielectric-graphenedielectric tunable infrared waveguide with ultrahigh refractive indices. Opt Express 21, 17089-17096 (2013)

14. Zhukovsky, S.V., Andryieuski, A., Sipe, J.E., Lavrinenko, A.V.: From surface to volume plasmons in hyperbolic metamaterials: general existence conditions for bulk high-k waves in metal-dielectric and graphene-dielectric multilayers. Phys. Rev. B 90, 155429 (2014)

15. Nefedov, I.S., Valaginnopoulos, C.A., Melnikov, L.A.: Perfect absorption in graphene multilayers. J. Opt. 15, 114003 (2013)
16. Andryieuski, A., Khromova, I., Zhukovsky, S.V., Lavrinenko, A.V.: GrapheneEnhanced Metamaterials for THz Applications. In: Fundamental and Applied Nano-Electromagnetics, pp. 145-169. Springer Netherlands, Dordrecht (2016)

17. Othman, M.A., Guclu, C., Capolino, F.: Graphene-dielectric composite metamaterials: evolution from elliptic to hyperbolic wavevector dispersion and the transverse epsilon-near-zero condition. J. Nanophotonics 7, 073089-073089 (2013)

18. Fan, S., Joannopoulos, J.D.: Analysis of guided resonances in photonic crystal slabs. Phys. Rev. B 65, 235112 (2002)

19. Berreman, D.W.: Optics in stratified and anisotropic media: $4 \times 4$-matrix formulation. Josa 62, 502-510 (1972)

\section{Submit your manuscript to a SpringerOpen ${ }^{\circ}$ journal and benefit from:}

- Convenient online submission

- Rigorous peer review

- Immediate publication on acceptance

- Open access: articles freely available online

- High visibility within the field

- Retaining the copyright to your article 